\title{
Therapeutic Strategies for Tendon Healing Based on Novel Biomaterials, Factors and Cells
}

\author{
Gerhard Gross $^{a} \quad$ Andrea Hoffmann ${ }^{b}$ \\ a Department of Molecular Biotechnology, Signalling and Gene Regulation, Helmholtz Centre for

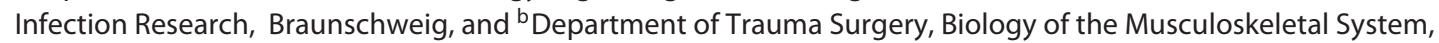 \\ Hannover Medical School, Hannover, Germany
}

\section{Key Words}

Tendon injury · Tissue regeneration · Bioscaffolds · Growth factor, cell and gene therapies

\begin{abstract}
The repair of tendon injuries still presents a major clinical challenge to orthopedic medicine. Tendons, like some other tissues, are poorly vascularized and heal slowly. In addition, healing often leads to the formation of fibrous tissue and scar tissue which lack flexibility and biomechanical properties. So the treatment of tendon injuries is challenging. We give an overview of the structure and composition of tendons, pathological states of tendon and natural healing, as well as therapeutic options. We focus in particular on biomaterials that have been specifically developed or suggested for the successful repair of tendon injuries. In addition, we also review factor-and cell-dependent strategies to heal tendon and ligament disorders. Although brief, we hope that this review will be helpful, particularly for those readers who are new to the field of tendon tissue engineering.
\end{abstract}

Copyright $\odot 2013$ S. Karger AG, Basel

\section{KARGER}

E-Mail karger@karger.com

www.karger.com/pat

\section{Structure and Composition of Tendons}

Ligaments and tendons, the flexible structures that bind together the musculoskeletal system, are extraordinarily strong in resisting tensile loads. Both are composed of dense fibrous connective tissue but they differ in morphology and function. Due to substantial biochemical differences, they also differ in their biomechanical properties. On average, tendons contain fewer cells and are less metabolically active than ligaments. The modest metabolic rate and well-developed generation of anaerobic energy of tendons and ligaments enable them to bear loads and maintain tension for extended periods of time.

A characteristic feature of tendons is the wavy crimp pattern that the collagen fibers demonstrate and which is seen on histology: in the absence of load, a regular sinusoidal wave pattern is observed in longitudinal sections. Upon stretching, the crimp pattern disappears and the fibers become straight. Upon release, the crimped pattern reappears. Tendons and ligaments exhibit a hierarchical structure which is based on the organization of collagen type I, a triple-helical molecule: The soluble tropocollagen molecules spontaneously self-assemble after secretion and cross-linking into collagen microfibrils. These
(C) 2013 S. Karger AG, Basel

$1015-2008 / 13 / 0804-0203 \$ 38.00 / 0$
Andrea Hoffmann

Department of Trauma Surgery, Biology of the Musculoskeletal System OE 8893 Hannover Medical School

Feodor-Lynen-Strasse 35, DE-30625 Hannover (Germany)

E-Mail hoffmann.andrea@mh-hannover.de 
microfibrils arrange themselves into larger units of subfibrils and fibrils. The fibrils then gather into collagen fibers that can be detected by light microscopy. Figures depicting the structural hierarchy of ligaments and tendons are depicted in a number of recent reviews to which we refer (see [1]).

The key difference between tendons and ligaments is their anatomical position (cf. below) rather than their histology or gene expression profile [2]: they express many similar proteins including extracellular matrix (ECM) and soluble proteins and transcription factors like TGF- $\beta$, scleraxis $(\mathrm{Scx})$, decorin and other proteoglycans, tenomodulin, fibromodulin, biglycan and the collagens [3]. Due to a lack of specific 'markers' many studies do not distinguish between these two tissues. To generate functional and self-renewing tendon tissue, the normal processes of tendon development must be elucidated in more detail. In particular, we need to understand which stem cell populations in the body are able to form tendons and ligaments, how they can be directed to do so in culture without simultaneously forming other skeletal tissues, how their growth is controlled and how normal cell turnover can be re-established and maintained in the tissueengineered tendon.

Ligaments connect bones to each other in order to restrict their relative motions and they also support organs. According to this definition, the patellar tendon is, in fact, a ligament. Tendons link muscles to bone and thus provide a connection which transmits forces developed by muscle contractions. On the one hand, a single tendon (e.g. the Achilles tendon) can focus the action of several muscles onto one bone. On the other hand, a single muscle (e.g. the tibialis posterior) can spread its action through several tendons that attach to different bones. Individual tendons vary a lot in shape, size and function, representing adaptations to specialized functions and this should be considered for therapeutic treatments. Tendons also function in proprioception. A third function of tendons is energy storage in form of elastic strain energy in stretched tendons.

Seventy percent of the tendon mass is water. Within the dry mass, the collagen content amounts to between 75 and $85 \%$, with $95 \%$ being type I collagen and $5 \%$ being type III and/or V. Their elastin content of less than 3\% dry weight is significantly lower than in ligaments, and proteoglycans, glycosaminoglycans and glycoproteins constitute about $2 \%$ [4]. Tenoblasts are immature tendon cells. They are spindle-shaped, with numerous cytoplasmic organelles reflecting their high metabolic activity. As they age, tenoblasts become elongated and transform into tenocytes. These have a lower nucleus-to-cytoplasm ratio than tenoblasts, with decreased metabolic activity, are interspersed between the collagen bundles and lie along the long axis of the tendon [5].

The structural hierarchy of tendons resembles that of ligaments but is more elaborate as collagen fibers are arranged in discrete packets called fibers or subfascicles (primary fiber bundles) and fascicles (secondary fiber bundles) that are bound together by the endotenon. The endotenon is surrounded by and continuous with the epitenon which surrounds the whole tendon. Surrounding the epitenon superficially, another thin layer called the paratenon is present which allows free movement within the surrounding tissue. Epitenon and paratenon together constitute the peritenon. Long tendons such as the digital flexor tendon that are present in areas subjected to increased mechanical stress - specifically in the hands and the feet - are additionally enclosed in a synovial sheath that gives lubrication and enhances gliding.

There are two junctions (attachment sites) for each tendon. These junctions need to resist forces of hundreds of Newtons and are also highly prone to injury. The different physical, structural/biochemical and mechanical properties of the tissues involved in the formation of these junctions need to be considered, particularly in the development of therapeutic options.

At the myotendinous junction, tendinous collagen fibrils are inserted into deep recesses formed by myocyte 'finger-like' processes, allowing the tension generated by intracellular contractile proteins of muscle fibers to be transmitted to the collagen fibrils. The folding of the tissues at the myotendinous junction strongly increases the contact area between the muscle and the tendon fibers and reduces the tensile stress exerted on the tendon during muscle contraction. Satellite cells (muscle stem cells) are present even in the adult myotendinous junction [6].

The osteotendinous junction (enthesis) occurs in two different shapes. A fibrocartilaginous (direct) enthesis (e.g. on the epiphyses of long bones or on the short bones of the wrist and ankle [7]) is composed of four zones: a dense fibrous connective tissue tendon zone, uncalcified fibrocartilage, mineralized fibrocartilage and bone. The outer border of calcification is indicated by a so-called tidemark with basophilic nature, similar to the tidemark found in articular cartilage. The tendon zone contains fibroblasts, whereas fibrocartilage cells prevail in the two fibrocartilage zones and osteoblasts/osteocytes in the bone. The fibrocartilage cells synthesize an ECM that is rich in aggrecan and collagen type II, both of which are typical of articular cartilage. Their function is to trap and 
hold water, thereby resisting compression and dissipating stress at the tendon-bone interface. As in articular cartilage, the fibrocartilage cells lie isolated within the matrix which creates a barrier to communication between the osteoblasts/osteocytes in the bone and the fibroblasts in the tendon [6]. A fibrous (indirect) enthesis (e.g. in the meta- or diaphyses of long bones [7]) lacks the fibrocartilage intermediate zone and is made up of tendon and bone zones only.

\section{Pathology and Natural Healing}

Tendon and ligament injuries account for considerable morbidity both in sport and in the workplace, and often prove disabling for several months. Chronic problems caused by overuse of tendons account for about $30 \%$ of all running-related injuries, and the prevalence of elbow tendinopathy in tennis players can be as high as $40 \%$ [5]. The social and economic burden associated with these medical conditions both in athletes and in working and elderly people calls for a greater understanding of basic tendon biology and expanding research on translational applications. Tendon injuries can be acute or chronic and are caused by intrinsic or extrinsic factors, either alone or in combination. Intrinsic factors are: age, gender, biomechanics, the presence or absence of systemic diseases and probably also genetic factors as discussed by Rees et al. [8]. Extrinsic factors include: physical load, environment, occupation and training. In acute trauma, extrinsic factors predominate, whilst in chronic cases, intrinsic factors also play a role. Overuse injuries generally have a multifactorial origin.

The terms 'tendonitis' or 'tendinitis' are a description of 'tendon inflammation' and as such are not very appropriate: although inflammatory conditions of the tendon are possible, they are not the principal cause of tendon injury and therefore, traditional treatment modalities aimed at controlling inflammation may not be optimal. Instead, the most common disorders involve degenerative processes due to an inability to resist the loads to which the tendon is subjected. Therefore, in general, these conditions are more of a degenerative than an inflammatory nature, and inflammatory reactions occur essentially only subsequent to the damage. Therefore, the term 'tendinosis' (a degenerative tendon without accompanying inflammation) to describe the tendon degeneration in tendinopathy (no implication for pathology is included in this term) would be more appropriate [8]. 'Tenosynovitis' implies imflammatory changes in synovial sheaths, but is a term commonly used in any tendon sheath disorder, particularly in the hand and wrist [9].

Certain tendons are especially vulnerable to degenerative pathology, including the Achilles, patella, parts of the rotator cuff (supraspinatus), forearm extensors, biceps brachii and tibialis posterior tendons. Injuries at the osteotendinous junction ('enthesopathies') can result from repetitive strains from metabolic, endocrine and inflammatory diseases. They are common, usually accompanied by muscle weakness and include tennis elbow, golfer's elbow and jumper's knee [9].

The natural healing process can be divided into three overlapping phases: first, the inflammatory stage within hours to a few days, second, the remodeling stage within a few days when tenocytes gradually migrate to the wound and type III collagen synthesis is initiated, and third, the modeling stage after about 6 weeks. Phase 3 can be divided into a consolidation and a maturation phase during which the healing tissue is resized and reshaped. The molecular events during these stages are well characterized. However, the use of selected growth factors or ECM molecules singly or in combination has not yielded notable therapeutic effects.

Although there is constant cell renewal and matrix turnover at a relatively slow rate in tendons, their hypovascularity, hypocellularity and the low metabolic rate entail slow healing after injury [10]. At the end of natural healing, the biochemical and mechanical properties never match those of intact tendons. Obviously, the cellular and molecular signals that lead to the formation of the native insertion site during embryogenesis and development are not recapitulated during tendon healing $[3,11]$, resulting in scar formation with collagen III prevailing. In addition, tendon damage is an important cause of successive joint instability and may progress into early onset of osteoarthritis, pain, disability and eventually the need for joint replacement surgery. Because the healing responses are different in ligaments and tendons after injury, the consequences and treatments are tissue- and site-specific. Nonsurgical managements like rest, compression, cooling, corticosteriod injection, ultrasound or laser treatment mainly provide pain relief. When these fail, surgery is required [3]. Surgical reconstructions including tissue autografts (using patellar or hamstring tendons), artificial prostheses or graft-augmentations are regularly performed by clinicians, but the long-term results are not satisfactory as the operated tissue is prone to reinjury besides the numerous complications accompanying surgeries [12]. In summary, the management of tendon injury continues to pose a considerable challenge because the 
current treatment options mainly allow for a replacement or a minimization of the tissue damage which results in pain relief or replacement of the pathological tissue but does not heal the damage itself. Fully functional tissue ('regeneration') is never achieved.

\section{Therapeutic Options}

Despite all efforts, current treatment modalities are not optimal, and alternative strategies are required. Our knowledge of the mechanisms regulating tendon development during embryogenesis is scarce. Even more limited is our knowledge concerning the molecular pathways that are involved in tendon regeneration in adults. In addition, there are clear limitations in defining the optimal conditions for tenogenic differentiation in vitro and the markers to assess a successful differentiation into tendonforming cells [3]. This complicates evaluation of in vitro and in vivo studies. To achieve scientific and clinical progress, a combination of knowledge derived from different scientific disciplines including molecular biology/ genetics, biochemistry, biomechanics, nanotechnology and engineering will advance the tissue engineering and regenerative medicine of tendons. These include: novel ECM ('biomimetic') bioscaffolds, the delivery of growth factors, (stem) cell-derived therapy, gene-therapeutic approaches based on vehicles encoding selected factors and advanced bioreactors with application of mechanical load. We review some recent developments in these topics. Although much of this work is promising, further investigations are necessary to achieve consistent results that can also be translated into clinical practice. As far as the use of cells and stem cells is concerned, regulatory issues may apply that further complicate routine clinical applications. At present, it is difficult to predict which of the individual strategies will have the most beneficial input in the future.

\section{Scaffolds, Biomimetic Materials and Grafts}

Depending on their origin, scaffolds can be fully natural, fully synthetic or a mixture of both. Another method of classification distinguishes resorbable/biodegradable from nonresorbable/permanent scaffolds depending on the chemical nature of the scaffold's components. The ideal scaffold should have some key properties that can be summarized by the following catchwords: biodegradability, biocompatibility, processability, mechanical strength and biofunctionality. In terms of biodegradability, the scaffold should provide temporary support until the neotissue has been built up into a state that will be able to function and bear loads. In vivo degradation will subse- quently obviate surgical removal. Biocompatibility means absence of an immunological response, interaction with and ideally integration into the host tissue. Processability implies the possibility of mimicking native tissue and addressing tissue needs. The scaffold should be able to bridge any complex 3-dimensional anatomical defect. Porous scaffolds enhance tissue regeneration by delivering biofactors. Pore diameter is important in facilitating cell migration, proliferation and growth factor movement. It is important to obtain the right balance between tissue regeneration and the mechanical properties of the scaffold. Whilst smaller pores are inefficient, larger pores can compromise these mechanical properties [13]. In addition, a scaffold should be easy to handle, store and sterilize.

Biological (natural) bioscaffolds consist of proteinbased extracellular matrices that are mammalian-derived tissues from human (allografts), equine, porcine and bovine (xenografts) sources $[14,15]$ such as the small intestinal submucosa (SIS, mostly from porcine jejunum), urinary bladder membrane, pericardium or dermis (TissueMend). They are processed to remove noncollagen components while retaining the natural collagen structure (predominantly collagen I fibers) and mechanical properties [15]. Materials that have been approved by the Food and Drug Administration (FDA) include Restore ${ }^{\mathrm{TM}}$ and CuffPatch (both from SIS), OrthADAPT ${ }^{\mathrm{TM}}$ (equine pericardium), Zimmer collagen repair patch and Permacol (both porcine acellular dermal matrix), Bio-BlanketW, TissueMend (bovine dermis and bovine dermal ECM) and GraftJacket (human acellular dermal matrix) [16]. The major drawback of these commercial biological scaffolds is that they have significantly less mechanical properties than normal tendons [17].

The collagen matrix ( $90 \%$ of dry weight) in SIS is immediately ready for graft purposes, and the extracellular proteins (elastin, laminins, fibronectins and proteoglycans) confer an additional layer of stability to the product. SIS also contains proteins like fibroblast growth factor (FGF) and transforming growth factor beta (TGF- $\beta$ ) which support healing. However, SIS has been shown to undergo contracture in vivo, and the high batch-to-batch variability limits the therapeutic potential. Harvesting SIS can elicit an immunological reaction and diminish its applicability for patients as an amplified inflammatory response can lead to tissue damage and poor wound healing.

Natural base scaffolds (based on natural polymers) have also been investigated in tendon repair: silk, fibrin or polymers including polysaccharides and proteins that 
share similar features with the natural ECM [17]. Silkcollagen hybrid scaffolds developed in a knitted sponge matrix have been described, as well as the application of alginate and chitosan hybrid fibers to support tendon fibroblast adhesion [3].

Silk-based biomaterials have obtained great attention lately as silk is a biodegradable and biocompatible material. Due to its biomechanical features, various promising silk-dependent strategies have been devised for clinical application [18] and as a scaffold for adult mesenchymal stem cells (MSCs) [19]. Fibrin has been suggested as another promising biomaterial as a matrix for tendon engineering [20] and a strategy to deliver stem cells [21] or growth factors [22]. Several other investigations emphasize the potential of collagen gels for tendon engineering [23-25], although the reproducibility of this therapeutic approach may cause problems due to collagen batch variations [26].

Synthetic scaffolds can have much stronger mechanical properties than natural scaffolds, but their biocompatibility is limited [15]. Nondegradable synthetic polymers with FDA approval for tendon repair include polyethylene terephthalate, polypropylene and poly(tetrafluoroethylene) [16]. Several biodegradable synthetic polymers have also obtained FDA approval. These include polylactic and polyglycolic acid, polycaprolactone and polydioxanone [16]. Several polymers (both nondegradable and degradable) may support growth factor release and therefore offer even greater flexibility. Although results from studies have been promising, scaffolds made of polyglycolic acid, for instance, have a limited application due to their mechanical brittleness and the lack of functional groups for signalling molecules. However, none of these synthetic scaffolds has exceeded the natural scaffolds in performance enough to make novel polymers an active field of research. One material that might be promising - poly(1,8-octanediol-co-citrate, POC) - has not yet obtained FDA approval. Synthetic polymers are, in general, more versatile than natural ones, enabling the tailoring and controlling of chemical and physical properties and structural features. They also represent a more reproducible source of raw materials and exhibit a low immunogenicity. The manipulation of structural parameters in the design of scaffolds and their bioactivation, through the incorporation of soluble and insoluble signals for promoting cell activities, is likely to improve the neoformation of tissues [3]. Knitting, braiding and electrospinning are the major techniques used for preparation of biomimetic fibrous scaffolds in tendon tissue engineering. When seeded with cells, they spontaneously orientate along the direction of the fibers, leading to abundant ECM secretion rich in collagens I and III [13].

The designation 'graft' applies to tissue that serves as a transplant. Such grafts can be used as an alternative to scaffolds or biomimetic materials, especially to replace severely damaged tendons. Patellar tendon and anterior cruciate ligament tissues are commonly used autografts for tendinopathies. Allografts are not as ideal due to the risk of disease transmission and rejection. Both autografts and allografts are retrieved from a nondiseased site elsewhere in the body which may lead to notable morbidities at the donor site.

\section{Delivery of Proteinaceous Factors}

Various growth factors, such as bFGF i.e. FGF-2, TGF- $\beta$ /BMPs (bone morphogenetic proteins), PDGF (platelet-derived growth factor), EGF (epidermal growth factor), VEGF (vascular endothelial growth factor), IGF (insulin-like growth factor), GDFs (growth and differentiation factors: mainly GDF5, GDF6 and GDF7 [11-13]) and HGF (hepatocyte growth factor), have been shown to be necessary for natural healing and have also demonstrated experimental usefulness for tendon repair.

Only a few transcription factors have been documented to be expressed specifically in tendon tissue and/or to contribute to tendon development. Scx expression is predominantly confined to tendons $[27,28]$. It has been shown, however, that tendon progenitor cells and tendons develop in Scx-mutant mice [29]. A potential role of Scx in tendon therapy, therefore, seems limited. Nevertheless, adenoviral-mediated Scx expression in MSCs seems to improve healing of tendon-bone attachment sites after rotator cuff repair [30]. The finding that Scx upregulates BMP4 expression in tendon cells at the site of tendon-bone insertions [31] could support this observation. Other investigations show that the homeodomain transcription factor Mohawk influences tendon morphogenesis [32]. In Drosophila, tendon differentiation has been attributed to the transcription factor Stripe. Its vertebrate homologues Egr1 and Egr2/Krox20 seem also to exert an influence upon tendon formation [33]. In addition, the constitutive active signaling factor Smad8 $(\mathrm{L}+\mathrm{MH} 2)$ seems to be able to support the formation of tendon-like tissue and the insertion of tendon into bone as well $[34,35]$. Due to the intracellular localization of all these proteins, a protein transduction domain for intracellular delivery of recombinant proteins would be needed. 
Due to their limited half-life in vivo, the direct, local delivery of growth factors has limited use, necessitating more advanced strategies for a sustained, safe and reproducible delivery. These include gene therapy, scaffolds, microspheres, microcapsules or nanocapsules as vehicles as well as coated sutures or dissolution in fibrin sealant [36]. Using appropriate measures, this will also allow for a controlled spatiotemporal release of factors and improve the long-term stability and storage of these factors in tailored systems. Several strategies have even aimed at the simultaneous delivery of several factors. The potential of using synthetic PLGA microspheres, fibrin-heparin delivery systems, metallic porous materials and so on, as well as the refinement of these systems, is being investigated [37]. The application of platelet-rich plasma as a source for various growth factors which efficiently contribute to anterior cruciate ligament healing $[38,39]$ is also promising, although platelet-rich plasma on its own seems to not be sufficient [40].

\section{Cell Sources for Therapeutic Strategies}

Autologous cells are likely to provide an optimal cell source, avoiding the immune reactions or complications caused by grafts. Their use may be limited by availability and accessibility including the risk of donor-site morbidity at the site of harvest. The techniques for their successful expansion in cell culture are still quite limited [41]. Tenocytes or fibroblasts isolated from the rotator cuff [42], patellar and Achilles tendons [20,43,44] or from the tendon sheath present an obvious choice [45] that is being investigated. Dermal fibroblasts are an alternative option $[24,46,47]$. They are commonly harvested by skin biopsy, which is a simple, quick and cost-effective procedure. They are easily expanded during in vitro culture. More importantly, they share characteristics with tenocytes, as both cell types are terminally differentiated cells and originate from mesoderm [3]. Despite common characteristics, however, it is questionable whether these terminally differentiated skin fibroblasts transdifferentiate into tenocytes in vivo. Such transdifferentiation may necessitate the expression of certain genes/factors similar to the reprogramming transcription factors used to generate induced pluripotent stem cells.

Clearly, pluripotent stem cells (induced pluripotent or embryonic) present an alternative choice. They will, however, necessitate the development of careful differentiation strategies towards tenocytes. As mentioned in the introductory paragraph, many molecular and phenotypical traits making up true tendon cells are unknown at present. Therefore, the development of such strategies will not be an easy task. In addition, the risks of teratoma formation must be excluded for pluripotent stem cells and ethical issues relating to the origin of the embryonic stem cells would also need clarification.

Therefore, a cell replacement therapy using alternative cell sources may appear reasonable. This may include other tissue-specific cells or stem cells. Adult stem cells open up the possibility to treat diverse diseases by autologous transplantation (i.e. using the patient's own cells after ex vivo expansion), either via local application or systemic infusion. Several studies using adult MSCs have been conducted in the last few years, especially in orthopedics as they have the potential to differentiate into cell lineages of the musculoskeletal system including tendon cells. In addition, MSCs secrete a variety of factors ('trophic' or 'paracrine actions') which help in their recruitment to sites of injury and subsequently in the regeneration of tissue by activation of endogenous (stem) cells. Bone marrow is the most popular source of MCSs due to its relative ease of access; alternatively, amniotic fluid, umbilical cord blood, adipose tissue, synovium and other tissues can serve as sources for the isolation of MSCs. Bone marrow-derived MSCs may have valid clinical utility, and various strategies have been used to coax these cells down a tenogenic lineage, including the application of growth factors, the ectopic expression of transcription factors, exposure to tensile loads and high-density coculture with tenocytes [36]. It is worth mentioning that tendon-derived stem/progenitor cells have been isolated and cultured from human, mouse, rabbit and rat tendons [48-51]. Their abundance, however, seems to be limited and their harvest is strongly invasive (as has been discussed above for tenocytes).

In summary, an ideal external cell source for tendon regeneration has not yet been identified and needs further investigation. In the light of regulatory issues already mentioned, it appears even more attractive to identify endogenous factors that would allow for a stimulation of endogenous cells to execute a tendon regeneration program.

\section{Future Aspects}

At present, there is still considerable room for improvement regarding the therapy of tendon injuries. Novel approaches involving the combination of mechanical stimuli, growth factors and/or hormones have to support tendon regeneration. Moreover, cell- and gene therapeutic strategies with tendon-derived cells or MSCs may 
eventually succeed in the regeneration of scar-free tendons and lead to biomechanical properties comparable with native tissue. Most important, however, improved scaffolds with an enhanced balance between stiffness and elastic compliance have to provide new therapeutic modalities for tendon disorders.

\section{Acknowledgements}

The authors gratefully acknowledge the support of the German Research Foundation (Deutsche Forschungsgemeinschaft) within the Collaborative Research Center SFB 599 (to G.G. and A.H.) and grant HO 2058/4-1 to A.H.

\section{References}

1 Hoffmann A, Gross G: Tendon and ligament engineering: from cell biology to in vivo application. Regen Med 2006;1:563-574.

2 Hasson P: 'Soft' tissue patterning: muscles and tendons of the limb take their form. Dev Dyn 2011;240:1100-1107.

>3 Rodrigues MT, Reis RL, Gomes ME: Engineering tendon and ligament tissues: present developments towards successful clinical products. J Tissue Eng Regen Med 2012, Epub ahead of print.

4 Martin RB, Burr DB, Sharkey NA: Skeletal Tissue Mechanics. New York, Springer, 1998.

5 Sharma P, Maffulli N: Basic biology of tendon injury and healing. Surgeon 2005;3:309-316.

6 6 Benjamin M, Ralphs JR: The cell and developmental biology of tendons and ligaments. Int Rev Cytol 2000;196:85-130.

7 Benjamin M, Qin S, Ralphs JR: Fibrocartilage associated with human tendons and their pulleys. J Anat 1995;187:625-633.

8 Rees JD, Wilson AM, Wolman RL: Current concepts in the management of tendon disorders. Rheumatology (Oxford) 2006;45:508521.

-9 Benjamin M, Ralphs JR: Tendons in health and disease. Man Ther 1996;1:186-191.

-10 Amiel D, Frank C, Harwood F, Fronek J, Akeson W: Tendons and ligaments: a morphological and biochemical comparison. J Orthop Res 1984;1:257-265.

11 Galatz LM, Rodeo SA: Editorial. J Shoulder Elbow Surg 2012;147-148.

$\checkmark 12$ Hsu SL, Liang R, Woo SL: Functional tissue engineering of ligament healing. Sports Med Arthrosc Rehabil Ther Technol 2010;2:12.

-13 Yates EW, Rupani A, Foley GT, Khan WS, Cartmell S, Anand SJ: Ligament tissue engineering and its potential role in anterior cruciate ligament reconstruction. Stem Cells Int 2012;2012:438125.

14 Badylak SF, Freytes DO, Gilbert TW: Extracellular matrix as a biological scaffold material: structure and function. Acta Biomater 2009;5:1-13.

15 Chen J, Xu J, Wang A, Zheng M: Scaffolds for tendon and ligament repair: review of the efficacy of commercial products. Expert Rev Med Devices 2009;6:61-73.

16 Thaker H, Sharma AK: Engaging stem cells for customized tendon regeneration. Stem Cells Int 2012;2012:309187.
17 Mano JF, Silva GA, Azevedo HS, Malafaya PB, Sousa RA, Silva SS, Boesel LF, Oliveira JM, Santos TC, Marques AP, Neves NM, Reis RL: Natural origin biodegradable systems in tissue engineering and regenerative medicine: present status and some moving trends. J R Soc Interface 2007;4:999-1030.

18 Fan H, Liu H, Toh SL, Goh JC: Anterior cruciate ligament regeneration using mesenchymal stem cells and silk scaffold in large animal model. Biomaterials 2009;30:4967-4977.

19 Teh TK, Toh SL, Goh JC: Aligned hybrid silk scaffold for enhanced differentiation of mesenchymal stem cells into ligament fibroblasts. Tissue Eng Part C Methods 2011;17:687-703.

20 Bayer ML, Yeung CY, Kadler KE, Qvortrup K, Baar K, Svensson RB, Magnusson SP, Krogsgaard M, Koch M, Kjaer M: The initiation of embryonic-like collagen fibrillogenesis by adult human tendon fibroblasts when cultured under tension. Biomaterials 2010;31: 4889-4897.

21 Hankemeier S, Hurschler C, Zeichen J, van GM, Miller B, Meller R, Ezechieli M, Krettek C, Jagodzinski M: Bone marrow stromal cells in a liquid fibrin matrix improve the healing process of patellar tendon window defects. Tissue Eng Part A 2009;15:1019-1030.

22 Thomopoulos S, Zaegel M, Das R, Harwood FL, Silva MJ, Amiel D, Sakiyama-Elbert S, Gelberman RH: PDGF-BB released in tendon repair using a novel delivery system promotes cell proliferation and collagen remodeling. J Orthop Res 2007;25:1358-1368.

23 Haddad-Weber M, Prager P, Kunz M, Seefried L, Jakob F, Murray MM, Evans $\mathrm{CH}$, Noth U, Steinert AF: BMP12 and BMP13 gene transfer induce ligamentogenic differentiation in mesenchymal progenitor and anterior cruciate ligament cells. Cytotherapy 2010;12:505-513.

24 Gigante A, Cesari E, Busilacchi A, Manzotti S, Kyriakidou K, Greco F, Di PR, Mattioli-Belmonte M: Collagen I membranes for tendon repair: effect of collagen fiber orientation on cell behavior. J Orthop Res 2009;27:826-832.

25 Awad HA, Butler DL, Boivin GP, Smith FN, Malaviya P, Huibregtse B, Caplan AI: Autologous mesenchymal stem cell-mediated repair of tendon. Tissue Eng 1999;5:267-277.

26 Friess W: Collagen - biomaterial for drug delivery. Eur J Pharm Biopharm 1998;45:113136.
27 Schweitzer R, Chyung JH, Murtaugh LC, Brent AE, Rosen V, Olson EN, Lassar A, Tabin CJ: Analysis of the tendon cell fate using scleraxis, a specific marker for tendons and ligaments. Development 2001;128:38553866.

28 Brent AE, Schweitzer R, Tabin CJ: A somitic compartment of tendon progenitors. Cell 2003;113:235-248.

29 Murchison ND, Price BA, Conner DA, Keene DR, Olson EN, Tabin CJ, Schweitzer R: Regulation of tendon differentiation by scleraxis distinguishes force-transmitting tendons from muscle-anchoring tendons. Development 2007;134:2697-2708.

30 Gulotta LV, Kovacevic D, Packer JD, Deng $\mathrm{XH}$, Rodeo SA: Bone marrow-derived mesenchymal stem cells transduced with scleraxis improve rotator cuff healing in a rat model. Am J Sports Med 2011;39:1282-1289.

31 Blitz E, Viukov S, Sharir A, Shwartz Y, Galloway JL, Pryce BA, Johnson RL, Tabin CJ, Schweitzer R, Zelzer E: Bone ridge patterning during musculoskeletal assembly is mediated through SCX regulation of Bmp4 at the tendon-skeleton junction. Dev Cell 2009;17:861873.

32 Liu W, Watson SS, Lan Y, Keene DR, Ovitt CE, Liu H, Schweitzer R, Jiang R: The atypical homeodomain transcription factor Mohawk controls tendon morphogenesis. Mol Cell Biol 2010;30:4797-4807.

33 Lejard V, Blais F, Guerquin MJ, Bonnet A, Bonnin MA, Havis E, Malbouyres M, Bidaud CB, Maro G, Gilardi-Hebenstreit P, Rossert J, Ruggiero F, Duprez D: EGR1 and EGR2 involvement in vertebrate tendon differentiation. J Biol Chem 2011;286:5855-5867.

34 Hoffmann A, Pelled G, Turgeman G, Eberle P, Zilberman Y, Shinar H, Keinan-Adamsky K, Winkel A, Shahab S, Navon G, Gross G, Gazit D: Neotendon formation induced by manipulation of the Smad8 signalling pathway in mesenchymal stem cells. J Clin Invest 2006;116:940-952.

35 Shahab-Osterloh S, Witte F, Hoffmann A, Winkel A, Laggies S, Neumann B, Seiffart V, Lindenmaier W, Gruber AD, Ringe J, Haupl T, Thorey F, Willbold E, Corbeau P, Gross G: Mesenchymal stem cell-dependent formation of heterotopic tendon-bone insertions (osteotendinous junctions). Stem Cells 2010;28: 1590-1601. 
36 Isaac C, Gharaibeh B, Witt M, Wright VJ, Huard J: Biologic approaches to enhance rotator cuff healing after injury. J Shoulder Elbow Surg 2012;21:181-190.

- 37 Anitua E, Sanchez M, Orive G, Andia I: Delivering growth factors for therapeutics. Trends Pharmacol Sci 2008;29:37-41.

38 Murray MM, Spindler KP, Abreu E, Muller JA, Nedder A, Kelly M, Frino J, Zurakowski D, Valenza M, Snyder BD, Connolly SA: Collagen-platelet rich plasma hydrogel enhances primary repair of the porcine anterior cruciate ligament. J Orthop Res 2007;25:81-91.

39 Joshi SM, Mastrangelo AN, Magarian EM, Fleming BC, Murray MM: Collagen-platelet composite enhances biomechanical and histologic healing of the porcine anterior cruciate ligament. Am J Sports Med 2009;37:24012410.

-40 Murray MM, Palmer M, Abreu E, Spindler KP, Zurakowski D, Fleming BC: Platelet-rich plasma alone is not sufficient to enhance suture repair of the ACL in skeletally immature animals: an in vivo study. J Orthop Res 2009; 27:639-645.
41 Scutt N, Rolf CG, Scutt A: Tissue specific characteristics of cells isolated from human and rat tendons and ligaments. J Orthop Surg Res 2008;3:32.

42 Pauly S, Klatte F, Strobel C, Schmidmaier G, Greiner S, Scheibel M, Wildemann B: Characterization of tendon cell cultures of the human rotator cuff. Eur Cell Mater 2010;20:8497.

43 Bernard-Beaubois K, Hecquet C, Houcine O, Hayem G, Adolphe M: Culture and characterization of juvenile rabbit tenocytes. Cell Biol Toxicol 1997;13:103-113.

44 Zhang J, Wang JH: Characterization of differential properties of rabbit tendon stem cells and tenocytes. BMC Musculoskelet Disord 2010;11:10

45 Liu W, Chen B, Deng D, Xu F, Cui L, Cao Y: Repair of tendon defect with dermal fibroblast engineered tendon in a porcine model. Tissue Eng 2006;12:775-788.

46 van Eijk F, Saris DB, Riesle J, Willems WJ, Van Blitterswijk CA, Verbout AJ, Dhert WJ: Tissue engineering of ligaments: a comparison of bone marrow stromal cells, anterior cruciate ligament, and skin fibroblasts as cell source. Tissue Eng 2004;10:893-903.
7 Tischer T, Vogt S, Aryee S, Steinhauser E, Adamczyk C, Milz S, Martinek V, Imhoff $\mathrm{AB}$ : Tissue engineering of the anterior cruciate ligament: a new method using acellularized tendon allografts and autologous fibroblasts. Arch Orthop Trauma Surg 2007;127: 735-741.

48 Bi Y, Ehirchiou D, Kilts TM, Inkson CA, Embree MC, Sonoyama W, Li L, Leet AI, Seo BM, Zhang L, Shi S, Young MF: Identification of tendon stem/progenitor cells and the role of the extracellular matrix in their niche. Nat Med 2007;13:1219-1227.

49 Rui YF, Lui PP, Li G, Fu SC, Lee YW, Chan KM: Isolation and characterization of multipotent rat tendon-derived stem cells. Tissue Eng Part A 2010;16:1549-1558.

50 Salingcarnboriboon R, Yoshitake H, Tsuji K, Obinata M, Amagasa T, Nifuji A, Noda M: Establishment of tendon-derived cell lines exhibiting pluripotent mesenchymal stem celllike property. Exp Cell Res 2003;287:289-300.

51 Zhang J, Li B, Wang JH: The role of engineered tendon matrix in the stemness of tendon stem cells in vitro and the promotion of tendon-like tissue formation in vivo. Biomaterials 2011;32:6972-6981. 